\title{
CCL3 and CXCL12 production in vitro by dental pulp fibroblasts from permanent and deciduous teeth stimulated by Porphyromonas gingivalis LPS
}

\author{
Carla Renata SIPERT ${ }^{1}$, Ana Carolina de Faria MORANDINI ${ }^{1}$, Karin Cristina da Silva MODENA ${ }^{2}$, Thiago José DIONÍSIO ${ }^{3}$, \\ Maria Aparecida Andrade Moreira MACHADO ${ }^{4}$, Sandra Helena Penha de OLIVEIRA ${ }^{5}$, Ana Paula CAMPANELLI \\ Carlos Ferreira SANTOS ${ }^{7}$
}

\author{
1- DDS, PhD, Department of Biological Sciences, Bauru School of Dentistry, University of São Paulo, Bauru, SP, Brazil. \\ 2- DDS, PhD, Department Operative Dentistry, Endodontics and Dental Materials, Bauru School of Dentistry, University of São Paulo, Bauru, SP, Brazil. \\ 3- MSc, Research Assistant, Department of Biological Sciences, Bauru School of Dentistry, University of São Paulo, Bauru, SP, Brazil. \\ 4- DDS, PhD, Professor, Department of Pediatric Dentistry, Orthodontics and Community Health; Bauru School of Dentistry, University of São Paulo, Bauru, \\ $\mathrm{SP}$, Brazil. \\ 5- PhD, Associate Professor, Department of Basic Sciences, Araçatuba School of Dentistry, Univ. Estadual Paulista - UNESP, Araçatuba, SP, Brazil. \\ 6- PhD, Associate Professor, Department of Biological Sciences, Bauru School of Dentistry, University of São Paulo, Bauru, SP, Brazil. \\ 7- DDS, PhD, Professor, Department of Biological Sciences, Bauru School of Dentistry, University of São Paulo, Bauru, SP, Brazil.
}

Corresponding address: Prof. Dr. Carlos F. Santos - Faculdade de Odontologia de Bauru - USP - Departamento de Ciências Biológicas - Disciplina de Farmacologia - Alameda Dr. Octavio Pinheiro Brisolla, 9-75 - Bauru - SP - 17012-901 - Brasil - e-mail: cebola@usp.br.

Received: January 18, 2013 - Modification: March 05, 2013 - Accepted: March 06, 2013

\section{ABSTRACT}

\begin{abstract}
bjective: The aim of this study was to compare the production of the chemokines CCL3 and CXCL12 by cultured dental pulp fibroblasts from permanent (PDPF) and deciduous (DDPF) teeth under stimulation by Porphyromonas gingivalis LPS (PgLPS). Material and Methods: Primary culture of fibroblasts from permanent $(n=3)$ and deciduous $(n=2)$ teeth were established using an explant technique. After the fourth passage, fibroblasts were stimulated by increasing concentrations of PgLPS $(0-10 \mu \mathrm{g} / \mathrm{mL})$ at 1,6 and $24 \mathrm{~h}$. The cells were tested for viability through MTT assay, and production of the chemokines CCL3 and CXCL12 was determined through ELISA. Comparisons among samples were performed using One-way ANOVA for MTT assay and Two-way ANOVA for ELISA results. Results: Cell viability was not affected by the antigen after $24 \mathrm{~h}$ of stimulation. PgLPS induced the production of CCL3 by dental pulp fibroblasts at similar levels for both permanent and deciduous pulp fibroblasts. Production of CXCL12, however, was significantly higher for PDPF than DDPF at 1 and $6 \mathrm{~h}$. PgLPS, in turn, downregulated the production of CXCL12 by PDPF but not by DDPF. Conclusion: These data suggest that dental pulp fibroblasts from permanent and deciduous teeth may present a differential behavior under PgLPS stimulation.
\end{abstract}

Key words: CCL3 chemokine. Chemokines. CXCL12 chemokine. Dental pulp. Fibroblasts. Dental pulp inflammation.

\section{INTRODUCTION}

Dental pulp is a loose mesenchymal tissue almost completely surrounded by a mineralized structure $^{5}$. Under normal conditions, dental pulp is sterile and primarily involved in the production of dentin and in tooth sensibility. When the dentin-pulp complex becomes infected by invading bacteria through caries or trauma, the pulpe tissue reacts in an attempt to eradicate these microorganisms ${ }^{23}$. Porphyromonas gingivalis $(\mathrm{Pg})$ has been found in deep caries and infected root canals, and may participate actively in pulpitis and periapical diseases development ${ }^{35,36}$. This microorganism presents virulence factors that are involved with innate immune response and inflammation progression, such as lipopolysaccharide (LPS) endotoxins $^{21}$. Special attention is being given to LPS, since the inflammatory and immune responses initiated by this antigen result from its recognition by membrane receptors such as CD14, Toll-like receptor (TLR) $4^{15}$ and TLR2 ${ }^{34}$. As a consequence, 
a cascade of intracellular signaling events is started, thus achieving the nuclear activation of proinflammatory genes ${ }^{15}$.

Since the innate immune system is activated by microbe associated molecular patterns (MAMPs), such as LPS, a wide variety of inflammatory mediators, growth factors and cytokines are produced in order to control the microbial infection?. Chemokines constitute an important family of cytokines, which are responsible for the trafficking of leukocytes resulting in the inflammatory infiltrate ${ }^{41}$. The classification of chemokines is based on their molecular structure concerning the position of the conserved cysteine residues (referred to as " $\mathrm{C}$ "). CC chemokines present adjacent cysteines, whereas CXC chemokines present cysteines separated by one amino acid (referred to as " $X$ ") ${ }^{41}$. The Macrophage Inflammatory Protein-1 $\alpha$ (MIP-1 $\alpha$, formally named $\mathrm{CCL} 3$ ) is a proinflammatory chemokine able to recruit monocytes, B lymphocytes, activated neutrophils ${ }^{3}$ and $\mathrm{CD}^{+}$and $\mathrm{CD}^{+}{ }^{+} \mathrm{T}$ lymphocytes ${ }^{31} \cdot \mathrm{CCL}^{3}$ is described as a chemokine able to orchestrate acute and chronic inflammatory events ${ }^{26}$. The Stromal cell Derived Factor (SDF)-1 (formally CXCL12) is a constitutive chemokine robustly produced by fibroblasts in normal conditions ${ }^{10}$. CXCL12 exerts chemotactic activity for lymphocytes, monocytes, neutrophils, immature dendritic cells ${ }^{29}$ and specially $\mathrm{CD} 4^{+}$stem cells ${ }^{20}$. Some recent data suggest that alterations in CXCL12 levels may result from the imbalance of tissue homeostasis ${ }^{10,20}$.

Fibroblasts are the most numerous cells in connective tissues. They are known as cells that not only provide structural support ${ }^{22}$, but may also function as accessory immune cells through antigen recognition and production of proinflammatory mediators including chemokines ${ }^{4}$. Although the ubiquitous distribution of fibroblasts along the entire human body, it has become increasingly evident that these cells present differences in phenotypic and functional characteristics depending on the anatomic site and pathologic status of their tissue of origin 4,13-14,32. Recent studies have shown differences in cytokines and chemokines production by fibroblasts from oral tissues such as gingiva and periodontal ligament ${ }^{13-14,27,32}$ or between gingival and skin fibroblasts ${ }^{2}$. However, little is known about possible differences regarding the production of chemokines between dental pulp fibroblasts from permanent and deciduous teeth.

Considering the importance of the knowledge about the immune response profile in the dental pulp connective tissue, we sought to investigate the in vitro production of the chemokines CCL3 and CXCL12 by cultured human permanent and deciduous dental pulp fibroblasts stimulated by Porphyromonas gingivalis LPS (PgLPS). Special attention was given to any potential differences in chemokines production between the tissue origin of these cells.

\section{MATERIAL AND METHODS}

\section{Primary culture of dental pulp fibroblasts}

All experiments were conducted in accordance with the Declaration of Helsinki. Ethical approval was obtained from the local Ethics Committee for Human Research (process \#15/2007). Prior to experiments, teeth were donated under patient informed consent. Human fibroblasts from dental pulp from permanent $(n=3)$ ( 1 male subject, aged 25 , and 2 female subjects, aged 18 and 22) and deciduous teeth $(n=2)$ ( 2 male subjects, aged 9 and 10 ) were cultured using an explant technique as described previously $27,28,33$. At the time of the dental extraction, all the teeth had indication for surgical removal. Permanent teeth did not present signs of inflammation or infection in the surrounding tissues, were totally erupted and presented closed apices. Deciduous teeth presented slight signals of physiological root resorption. The pulp tissue was removed in aseptic conditions, and after minced the fragments were incubated for cell growth in Dulbecco's modified Eagle's medium (DMEM) (Gibco, Invitrogen Corporation, Carlsbad, USA) supplemented with $10 \%$ fetal bovine serum (FBS; Gibco) and antibiotics (100 $\mathrm{gg} / \mathrm{mL}$ penicillin, 100 $\mu \mathrm{g} / \mathrm{mL}$ streptomycin, $0.5 \mathrm{mg} / \mathrm{mL}$ amphotericin B - Invitrogen Corporation, Carlsbad, USA). Cultures were maintained at $37^{\circ} \mathrm{C}$ in a humidified atmosphere of $5 \% \mathrm{CO}_{2}$ and $95 \%$ air. Cells were used between the fourth and eighth passages.

\section{Phenotypic characterization of fibroblasts}

Pulp cells were characterized as fibroblasts by their morphology and staining for procollagen $\mathrm{I}^{1}$ and fibroblast surface protein ${ }^{30}$ by means of immunostaining. Fibroblasts from permanent and deciduous teeth were seeded over sterile 12 $\mathrm{mm}$ round cover slips (BD Biosciences, Bedford, USA). After $24 \mathrm{~h}$ to allow cellular attachment, the cover slips were removed and fixed in acetone (Merck Chemicals, Darmstadt, Germany). Immunostaining was performed by using goat anti-human procollagen type I 1:100 (catalog \#sc-25973; Santa Cruz Biotechnology, Santa Cruz, USA) or mouse anti-human fibroblast surface protein (catalog \#ab11333, Abcam, Cambridge, UK) followed by rabbit anti-goat IgG fluoresceine conjugated antibody 1:200 (catalog \#FI-5000; Vector Labs., Burlingame, USA) or rabbit antimouse fluoresceine conjugated antibody 1:400 (catalog \#ab97045; Abcam), respectively. Slides were then mounted with a mounting medium containing DAPI (4',6-diamidino-2-phenylindole dihydrochloride hydrate) (catalog \#H-1200; Vector 
Labs.), thus allowing nuclear staining. Images were captured by an inverted confocal microscope (Leica TCS-SPE, Leica, Manheim, Germany).

\section{Cell viability}

Dental pulp fibroblasts from a permanent $(n=1)$ and a deciduous tooth $(\mathrm{n}=1)$ were detached, counted and seeded at a concentration of $1.25 \mathrm{x}$ $10^{4}$ cells/well in 96-well plates. After $18 \mathrm{~h}$ to allow cellular attachment, PgLPS (catalog code: tIrl-pglps; Invivogen, San Diego, USA) was added to the culture medium at concentrations ranging from 0 to $10 \mu \mathrm{g} /$ $\mathrm{mL}$ in triplicates. MTT [3-(4,5-Dimethylthiazol2-yl)-2,5-diphenyltetrazolium bromide] (catalog \# M2128, Sigma-Aldrich, St Louis, EUA) assay was performed after $24 \mathrm{~h}$. Cell supernatant was discarded, and $20 \mu \mathrm{L}$ of an MTT solution ( $5 \mathrm{mg} / \mathrm{mL}$ ) in phosphate buffered saline (PBS) were added to the cells or cell-free blank wells followed by 180 $\mu \mathrm{L}$ of $10 \%$ FBS DMEM. Cells were incubated for $4 \mathrm{~h}$ at $37^{\circ} \mathrm{C}$ with $5 \% \mathrm{CO}_{2}, 95 \%$ air. MTT solution was then removed and replaced with $100 \mu \mathrm{L}$ of isopropanol. The plate was incubated for $20 \mathrm{~min}$ at room temperature and the optical density (OD) of the wells was determined using a plate reader (FLUOstar Optima, BMG Labtech, Ortenberg, Germany) at $570 \mathrm{~nm}$ wavelength.

\section{Chemokines detection in fibroblasts supernatants}

Dental pulp fibroblasts from permanent $(n=3)$ and deciduous $(n=2)$ (DDPF) teeth were detached, counted and seeded at $5 \times 10^{4}$ cells/well in 24-well plates. After $18 \mathrm{~h}$ to allow cell attachment, PgLPS $(0.1-10 \mu \mathrm{g} / \mathrm{mL})$ or culture medium only was added to the cells in duplicate. After 1, 6 and 24 h cell supernatants were collected for chemokines detection. For one of the established DDPF culture, the experiment was conducted twice in order to obtain the final $n=6$ for both fibroblasts subtypes.

The production of CCL3 and CXCL12 was detected by means of enzyme-linked immunosorbent assay (ELISA) (Anti-human CCL3/MIP-1 $\alpha$ Antibody AF-270-NA and Biotinylated Anti-human CCL3/ MIP-1a Antibody - BAF 270, Monoclonal Antihuman/mouse CXCL12/SDF-1 Antibody - MAB 350 and Biotinylated Anti-human CXCL12/SDF-1 Antibody - BAF 310, R\&D Systems, Minneapolis, USA, respectively) according to the manufacturer's instructions.

\section{Statistical Analysis}

Statistical analysis was performed using Graph Pad Prism 5.0 (GraphPad Software, La Jolla, USA). One-way ANOVA was applied to the sets of data resulting from viability assay and Twoway ANOVA was performed for ELISA resulting data. Comparisons between pairs of groups were performed using Tuckey post-test. The significance level was set at $p<0.05$.

\section{RESULTS}

Phenotypic characterization of fibroblasts

Dental pulp fibroblasts were isolated and cultured as previously described ${ }^{33}$. Based on the results obtained by immunofluorescence, these cells were positive for procollagen I and fibroblast surface protein indicating their mesenchymal origin (Figure 1). Interestingly, DDPF presented higher fluorescence intensity for procollagen I in comparison to PDPF.
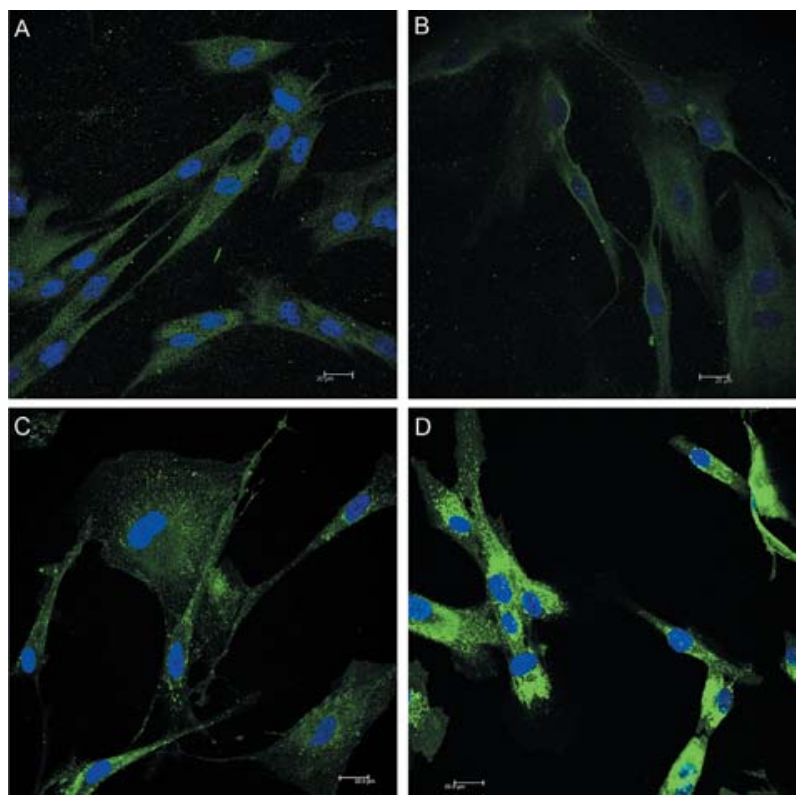

Figure 1- Characterization of dental pulp fibroblasts by procollagen I and fibroblast surface protein staining. Cultured dental pulp fibroblasts from permanent $(n=1)$ and deciduous $(n=1)$ teeth were immunostained for procollagen I ( $A$ and $B$ ) or fibroblast surface protein ( $C$ and D). A and C: PDPF; B and D: DDPF. Images captured by a confocal microscope (representative bars: $20 \mu \mathrm{m}$ ).
A

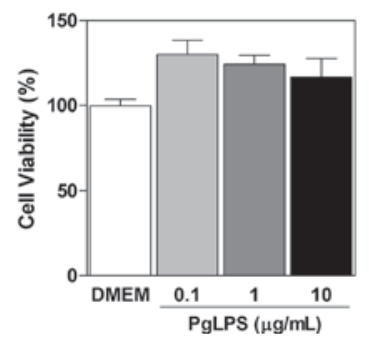

B

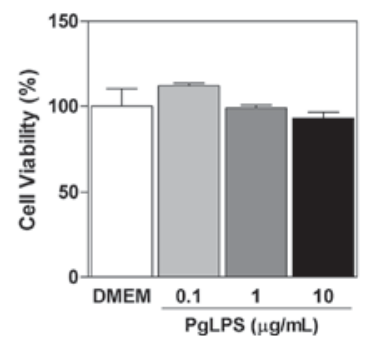

Figure 2- Cell viability of dental pulp fibroblasts challenged with $P$. gingivalis LPS. Cultured dental pulp fibroblasts from permanent $(n=1$; panel $A)$ and deciduous $(n=1$, panel $B$ ) teeth were stimulated by $P$. gingivalis LPS (PgLPS) at the indicated concentrations in triplicate. Cell viability was assessed by means of MTT assay $(n=3)$. 

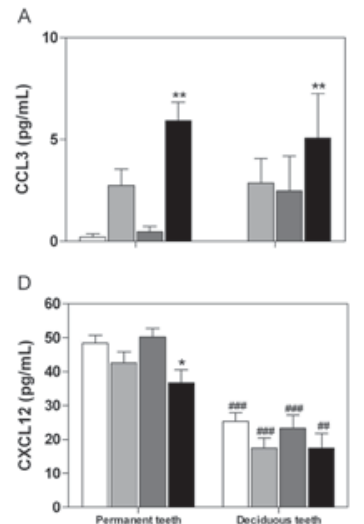

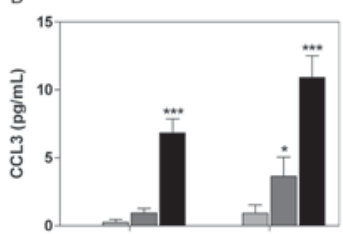

$\mathrm{E}$

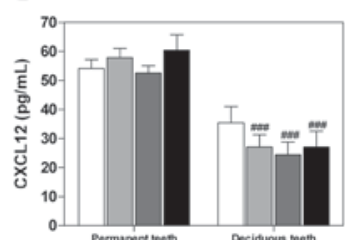

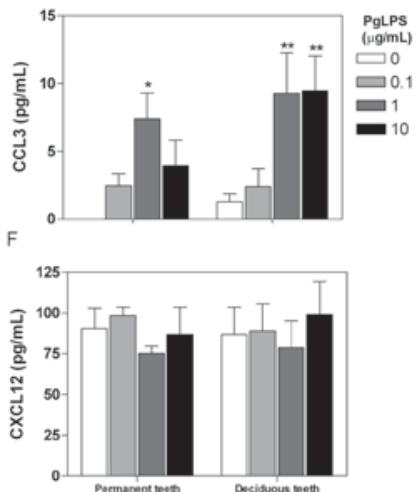

Figure 3- Production of chemokines by different subtypes of dental pulp fibroblasts. Cultured dental pulp fibroblasts from permanent $(n=3)$ and deciduous $(n=2)$ teeth were stimulated by $P$. gingivalis LPS at the indicated concentrations $(n=6)$. Cell supernatants were collected after 1 ( $A$ and $D), 6$ (B and E) and 24 (C and F) h. Production of CCL3 (A, B and C) and CXCL12 (D, E and F) was detected by means of ELISA. $\left({ }^{*}\right) p<0.05 ;\left({ }^{* *}\right) p<0.01$ and $\left({ }^{* * *}\right) p<0.001$ in comparison with culture medium alone (0). (\#\#) $p<0.01$ and (\#\#\#) $p<0.001$ in comparison with the other cellular subtype at the same experimental condition.

\section{Cell viability}

Cell viability of both permanent and deciduous dental pulp fibroblasts was not affected by PgLPS at any concentration during $24 \mathrm{~h}$ in comparison with medium only for PDPF (panel A) and for DDPF (panel B) (Figure 2; $p=0.0977$ and $p=0.1903$, respectively).

\section{Chemokines detection}

The chemokines CCL3 and CXCL12 were detected at the cell supernatants of permanent and deciduous dental pulp fibroblasts as observed in Figure 3.

PgLPS was able to induce CCL3 production for both permanent and deciduous dental pulp fibroblasts at $1 \mathrm{~h}(\mathrm{p}=0.0009), 6 \mathrm{~h}(\mathrm{p}<0.0001)$ and $24 \mathrm{~h}(p=0.0005)$. CCL3 increase was more evident at $10 \mu \mathrm{g} / \mathrm{mL}$ of PgLPS (Figure 3: A, B and C). The levels of this chemokine for both cellular subtypes were quite similar $(p=0.7682$ and 0.1115 at 1 and $24 \mathrm{~h}$, respectively). At $6 \mathrm{~h}$, a significant difference was found among PDPF and DDPF $(p=0.0089)$.

Production of CXCL12 was altered by the antigen only in PDPF at $10 \mu \mathrm{g} / \mathrm{mL}$ at $1 \mathrm{~h}$ in comparison with untreated cells $(p=0.0055)$. PgLPS was unable to alter CXCL12 production by DDPF, although these cells produced significantly lower CXCL12 levels in comparison with PDPF $(p<0.0001)$. At 6 h, CXCL12 production was not affected by the presence of the antigen, and DDPF produced significantly lower CXCL12 levels when compared to PDPF $(p<0.0001)$. Similar levels of this chemokine were observed for both fibroblasts subpopulations at $24 \mathrm{~h}(\mathrm{p}=0.9537)$ without significant alterations induced by PgLPS $(p=0.6546)$.

\section{DISCUSSION}

The findings observed in this study showed that dental pulp fibroblasts from permanent and deciduous teeth were able to produce in vitro the chemokines CCL3 and CXCL12. Upon immunological challenge by PgLPS, production of CCL3 by both cell types was significantly increased. On the other hand, the antigen affected the basal CXCL12 production only for pulp fibroblasts from deciduous teeth, which in turn produced higher levels of this chemokine.

Bacterial components may display an important role in the development of dental pulp inflammation since pulpal disease is initiated long before dental pulp tissue exposure to bacteria occurs ${ }^{8}$. LPS is one of best characterized bacterial cell wall components. Considering the existence of $P$. gingivalis in dental caries and its importance in symptomatic inflammatory processes ${ }^{12,18}$; the LPS of this microorganism was chosen in this study as the antigenic stimulus to the dental pulp fibroblasts.

Fibroblasts express innate immune receptors and related signaling molecules, including dental pulp ones ${ }^{16}$. TLR4 is the major membrane receptor involved in the process of LPS recognition by the host, including PgLPS sensing by human gingival fibroblasts ${ }^{37}$. Unlike Escherichia coli LPS, PgLPS structure presents an additional and particular antigenic portion in its molecule described as a lipoprotein that is suggested to be recognized by TLR2, which in turn is responsible for the recognition of cell wall components of gram-positive bacteria, such as lipotheichoic acids ${ }^{19}$. The role of TLR2 in PgLPS recognition has been recently supported ${ }^{9,34}$. Taken this together, it might be assumed that the cellular mechanisms involved in PgLPS recognition may be quite similar to biological events occurring 
in polimicrobial infections, such as dental caries.

To the best of our knowledge, this is the first report of the production of a chemokine by dental pulp fibroblasts in response to PgLPS. Previous studies showed the upregulation of the expression of $\mathrm{IL}-6^{40}$ and $\mathrm{IL}-8^{39}$ by $P$. gingivalis supernatant in dental pulp fibroblasts. In response to PgLPS, human gingival fibroblasts showed an upregulation of IL- $8^{24}$ and Interleukin IL-6 production ${ }^{38}$. PgLPS was also demonstrated to downregulate the production of CXCL12 by gingival ${ }^{17,27}$ and periodontal ligament fibroblasts ${ }^{27}$. In a previous study by our group, besides the alterations observed in CXCL12 production, PgLPS was able to induce CCL3 production by gingival fibroblasts, but not by periodontal ligament ones, and to induce the production of IL- 6 by both cellular types ${ }^{27}$. Production of proinflammatory mediators including cytokines and chemokines by fibroblasts may be of some clinical importance. Differently from peripheral blood monocytes (PBMC), human gingival fibroblasts were efficient to keep producing IL-6 after a pretreatment with PgLPS, which in turn abrogate IL- 6 production by PBMC in a secondary exposition to the antigen. Such study demonstrates that gingival fibroblasts do not present LPS tolerance as observed in PBMC2.

As mentioned before, fibroblasts may be considered a heterogeneous population among different anatomical regions ${ }^{4,14,27,32}$. Comparing dental pulp and gingival fibroblasts, Chu, et al. ${ }^{6}$ (2004) demonstrated a more effective induction of Vascular Endothelial Growth Factor mRNA in dental pulp fibroblasts by tumor necrosis factor (TNF)- $\alpha$ in comparison with gingival ones. Focusing on extracellular matrix expression, Martinez and Araújo $^{25}$ (2004) demonstrated positive staining for tenascin and osteonectin only in dental pulp fibroblasts, although the expression of fibronectin and collagen I could be detected both in gingival and dental pulp fibroblasts.

The present study showed for the first time that dental pulp fibroblasts from permanent teeth produced higher levels of the chemokine CXCL12 during early experimental periods ( 1 and $6 \mathrm{~h}$ ) in comparison with the cells derived from deciduous teeth. Distinct levels of chemokines may occur due to the fact that the expression of Toll-like receptor (TLR) 2 and TLR4 may vary in fibroblasts according to the anatomic site as well as CD14 and myeloid differentiation (MD)-2, molecules also actively involved in LPS recognition ${ }^{14}$. Additionally, CXCL12 production by fibroblasts may occur in response to other chemokines or cytokines such as TNF- $\alpha$, Interferon (IFN)- $\gamma$, Tranforming Growth Factor (TGF)- $\beta 1$, CCL5 (Regulated upon Activation Normal T-cell Expressed and Secreted - RANTES), CCL20 (MIP-3 $\alpha$ ) and $\mathrm{CX}_{3} \mathrm{CL} 1$ (Fractalkine) ${ }^{17}$. The levels of these regulating factors may also be produced in unique patterns by each cellular type. Taken this together, the precise cellular mechanisms that lead to the variable levels of CXCL12 produced by PDPF and DDPF observed in this study are probably complex and unpredictable based on the available scientific findings. Future studies are needed to understand the cellular and molecular mechanisms involved in potential phenotypic and functional differences presented by fibroblasts from permanent and deciduous dental pulps.

Differences in CXCL12 production may be of some biological importance considering evidence that points to the role of this chemokine in stem cells recruitment after tissue injury ${ }^{20}$. Also, the dynamics of CXCL12 production during the repair process following inflammation, condition that may lead to tissue CXCL12 decrease ${ }^{10}$, may differently affect permanent and deciduous dental pulps according to the present data.

PgLPS induced both PDPF and DDPF to produce similar levels of the chemokine CCL3. Production of CCL3 by pulmonary fibroblasts was described by Enzerink, et al.7 (2009) when these cells were arranged in a spheroid conformation instead of monolayer cultures. As abovementioned, in a previous study, our group showed the upregulation of CCL3 by PgLPS-stimulated gingival fibroblasts ${ }^{27}$. The present findings corroborate the data that suggest that fibroblasts are active cells during immune response activities, including the ones originally from deciduous dental pulps. In this field, our data corroborate Ferreira, et al. ${ }^{11}$ (2008), who showed that DDPF were able to produce IL-1 $\beta$ and IL-8 when stimulated by $E$. coli LPS and also by some dental materials.

\section{CONCLUSION}

PgLPS induced the production of CCL3 by dental pulp fibroblasts at similar levels for both permanent and deciduous pulp. Production of CXCL12 was significantly higher for PDPF than DDPF. These data suggest that dental pulp fibroblasts from permanent and deciduous teeth may present a differential behavior under inflammatory conditions.

\section{ACKNOWLEDGEMENTS}

The authors would like to demonstrate their gratitude to Gentília Borges Carvalho Tavares for her outstanding contribution during sample collection. This research was supported by the São Paulo Research Foundation (FAPESP) by means of Research Grant to CF Santos (process \#2005/60167-0) and Doctorate Scholarship to CR Sipert (process \#2007/00306-1). 


\section{REFERENCES}

1- Abd-Elmeguid A, Yu DC, Kline LW, Moqbel R, Vliagoftis $H$ Dentin matrix protein-1 activates dental pulp fibroblasts. J Endod. 2012;38(1):75-80.

2- Ara T, Kurata K, Hirai K, Uchihashi T, Uematsu T, Imamura Y, et al. Human gingival fibroblasts are critical in sustaining inflammation in periodontal disease. J Periodontal Res. 2009;44(1):21-7.

3- Bonecchi R, Polentarutti N, Luini W, Borsatti A, Bernasconi S, Locati $M$, et al. Up-regulation of CCR1 and CCR3 and induction of chemotaxis to CC chemokines by IFN-gamma in human neutrophils. J Immunol. 1999;162(1):474-9.

4- Brouty-Boye D, Pottin-Clemenceau C, Doucet C, Jasmin C, Azzarone B. Chemokines and CD40 expression in human fibroblasts. Eur J Immunol. 2000;30(3):914-9.

5- Chiego Jr. D. Histology of the pulp. In: Avery J, editor. Oral development and histology. $3^{\text {rd }}$ ed. ed. Stuttgart: Georg Thieme Verlag; 2002. p. 190-212.

6- Chu SC, Tsai CH, Yang SF, Huang FM, Su YF, Hsieh YS, et al. Induction of vascular endothelial growth factor gene expression by proinflammatory cytokines in human pulp and gingival fibroblasts. J Endod. 2004;30(10):704-7.

7- Enzerink A, Salmenpera P, Kankuri E, Vaheri A. Clustering of fibroblasts induces proinflammatory chemokine secretion promoting leukocyte migration. Mol Immunol. 2009;46(89):1787-95.

8- Farges JC, Keller JF, Carrouel F, Durand SH, Romeas A, Bleicher $F$, et al. Odontoblasts in the dental pulp immune response. J Exp Zool B Mol Dev Evol. 2009;312B(5):425-36.

9- Faria Morandini AC, Chaves Souza PP, Ramos-Junior ES, Brozoski DT, Sipert CR, Souza Costa CA, et al. Toll-like receptor 2 knockdown modulates interleukin (IL)- 6 and IL- 8 but not stromal derived factor-1 (SDF-1/CXCL12) in human periodontal ligament and gingival fibroblasts. J Periodontol. 2013;84(4):535-44

10- Fedyk ER, Jones D, Critchley HO, Phipps RP, Blieden TM, Springer TA. Expression of stromal-derived factor-1 is decreased by IL-1 and TNF and in dermal wound healing. J Immunol. 2001;166(9):5749-54.

11- Ferreira DC, Brito DG, Cavalcanti BN. Cytokine production from human primary teeth pulp fibroblasts stimulated by different pulpotomy agents. J Dent Child (Chic). 2009;76(3):194-8.

12- Ferreira DC, Rocas IN, Paiva SS, Carmo FL, Cavalcante FS, Rosado AS, et al. Viral-bacterial associations in acute apical abscesses. Oral Surg Oral Med Oral Pathol Oral Radiol Endod. 2011;112(2):264-71.

13- Ghosh A, Park JY, Fenno C, Kapila YL. Porphyromonas gingivalis, gamma interferon, and a proapoptotic fibronectin matrix form a synergistic trio that induces $\mathrm{c}$-Jun $\mathrm{N}$-terminal kinase 1-mediated nitric oxide generation and cell death. Infect Immun. 2008;76(12):5514-23.

14- Hatakeyama J, Tamai R, Sugiyama A, Akashi S, Sugawara S, Takada $\mathrm{H}$. Contrasting responses of human gingival and periodontal ligament fibroblasts to bacterial cell-surface components through the CD14/Toll-like receptor system. Oral Microbiol Immunol. 2003;18(1):14-23.

15- Hennessy EJ, Parker AE, O'Neill LA. Targeting Toll-like receptors: emerging therapeutics? Nat Rev Drug Discov. 2010;9(4):293-307.

16- Hirao K, Yumoto H, Takahashi K, Mukai K, Nakanishi T, Matsuo T. Roles of TLR2, TLR4, NOD2, and NOD1 in pulp fibroblasts. ] Dent Res. 2009;88(8):762-7.

17- Hosokawa Y, Hosokawa I, Ozaki K, Nakae H, Murakami $\mathrm{K}$, Miyake $\mathrm{Y}$, et al. CXCL12 and CXCR4 expression by human gingival fibroblasts in periodontal disease. Clin Exp Immunol. 2005; 141(3):467-74.

18- Jacinto RC, Gomes BP, Shah HN, Ferraz CC, Zaia AA, Souza-Filho FJ. Incidence and antimicrobial susceptibility of Porphyromonas gingivalis isolated from mixed endodontic infections. Int Endod J. 2006;39(1):62-70.
19- Jin MS, Kim SE, Heo JY, Lee ME, Kim HM, Paik SG, et al. Crystal structure of the TLR1-TLR2 heterodimer induced by binding of a tri-acylated lipopeptide. Cell. 2007;130(6):1071-82.

20- Karin N. The multiple faces of CXCL12 (SDF-1alpha) in the regulation of immunity during health and disease. J Leukoc Biol. 2010;88(3):463-73.

21- Koga T, Nishihara T, Fujiwara T, Nisizawa T, Okahashi N, Noguchi $\mathrm{T}$, et al. Biochemical and immunobiological properties of lipopolysaccharide (LPS) from Bacteroides gingivalis and comparison with LPS from Escherichia coli. Infect Immun. 1985;47(3):638-47.

22- Kuo MY, Lan WH, Lin SK, Tsai KS, Hahn LJ. Collagen gene expression in human dental pulp cell cultures. Arch Oral Biol. 1992;37(11):945-52.

23- Love RM, Jenkinson HF. Invasion of dentinal tubules by oral bacteria. Crit Rev Oral Biol Med. 2002;13(2):171-83.

24- Mahanonda R, Sa-Ard-Iam N, Montreekachon P, Pimkhaokham A, Yongvanichit K, Fukuda MM, et al. IL-8 and IDO Expression by Human Gingival Fibroblasts via TLRs. J Immunol. 2007;178(2):1151-7.

25- Martinez EF, Araujo VC. In vitro immunoexpression of extracellular matrix proteins in dental pulpal and gingival human fibroblasts. Int Endod J. 2004;37(11):749-55.

26- Maurer M, von Stebut E. Macrophage inflammatory protein-1. Int J Biochem Cell Biol. 2004;36(10):1882-6.

27- Morandini AC, Sipert CR, Gasparoto TH, Greghi SL, Passanezi $E$, Rezende $M L$, et al. Differential production of macrophage inflammatory protein-1alpha, stromal-derived factor-1, and IL-6 by human cultured periodontal ligament and gingival fibroblasts challenged with lipopolysaccharide from $P$. gingivalis. J Periodontol. 2010;81(2):310-7.

28- Morandini AC, Sipert CR, Ramos-Junior ES, Brozoski DT, Santos CF. Periodontal ligament and gingival fibroblasts participate in the production of TGF-beta, interleukin (IL)-8 and IL-10. Braz Oral Res. 2011;25(2):157-62.

29- Ricart BG, John B, Lee D, Hunter CA, Hammer DA. Dendritic cells distinguish individual chemokine signals through CCR7 and CXCR4. J Immunol. 2011;186(1):53-61.

30- Ronnov-Jessen L, Celis JE, Van Deurs B, Petersen OW. A fibroblast-associated antigen: characterization in fibroblasts and immunoreactivity in smooth muscle differentiated stromal cells. J Histochem Cytochem. 1992;40(4):475-86.

31- Schall TJ, Bacon K, Camp RD, Kaspari JW, Goeddel DV. Human macrophage inflammatory protein alpha (MIP-1 alpha) and MIP-1 beta chemokines attract distinct populations of lymphocytes. J Exp Med. 1993;177(6):1821-6.

32- Scheres N, Laine ML, de Vries TJ, Everts V, van Winkelhoff AJ. Gingival and periodontal ligament fibroblasts differ in their inflammatory response to viable Porphyromonas gingivalis. J Periodontal Res. 2010;45(2):262-70.

33- Sipert CR, Moraes IG, Bernardinelli N, Garcia RB, Bramante $\mathrm{CM}$, Gasparoto TH, et al. Heat-killed Enterococcus faecalis alters nitric oxide and CXCL12 production but not CXCL8 and CCL3 production by cultured human dental pulp fibroblasts. J Endod. 2010;36(1):91-4.

34- Souza PP, Palmqvist P, Lundgren I, Lie A, Costa-Neto CM, Lundberg $P$, et al. Stimulation of IL- 6 cytokines in fibroblasts by toll-like receptors 2. J Dent Res. 2010;89(8):802-7.

35- Sundqvist G, Johansson E, Sjogren U. Prevalence of blackpigmented bacteroides species in root canal infections. J Endod. 1989;15(1):13-9.

36- van Winkelhoff AJ, Carlee AW, de Graaff J. Bacteroides endodontalis and other black-pigmented Bacteroides species in odontogenic abscesses. Infect Immun. 1985;49(3):494-7.

37- Wang PL, Azuma Y, Shinohara M, Ohura K. Toll-like receptor 4-mediated signal pathway induced by Porphyromonas gingivalis lipopolysaccharide in human gingival fibroblasts. Biochem Biophys Res Commun. 2000;273(3):1161-7. 
38- Wang PL, Shirasu S, Shinohar M, Azuma Y, Daito M, Yasuda $\mathrm{H}$, et al. IL-10 inhibits Porphyromonas gingivalis LPS-stimulated human gingival fibroblasts production of IL-6. Biochem Biophys Res Commun. 1999;263(2):372-7.

39- Yang LC, Huang FM, Lin CS, Liu CM, Lai CC, Chang YC. Induction of interleukin-8 gene expression by black-pigmented Bacteroides in human pulp fibroblasts and osteoblasts. Int Endod J. $2003 ; 36(11): 774-9$.
40- Yang LC, Tsai CH, Huang FM, Liu CM, Lai CC, Chang YC. Induction of interleukin- 6 gene expression by pro-inflammatory cytokines and black-pigmented Bacteroides in human pulp cell cultures. Int Endod J. 2003;36(5):352-7.

41- Zlotnik A, Yoshie O. Chemokines: a new classification system and their role in immunity. Immunity. 2000;12(2):121-7. 